\title{
De l'origine des catastrophes : phénomènes extrêmes ou âpreté du quotidien?
}

\author{
Jean-Christophe Gaillard
}

Géographe, Université Joseph Fourier - Grenoble 1, Institut de géographie alpine, UMR5194 PACTE, 14 bis avenue Marie Reynoard, 38100 Grenoble, France

\begin{abstract}
La fonction d'un éditorial n'est-elle pas, en même temps que le rappel d'une ligne «éditoriale », un appel à échange et à discussion, voire une invitation au débat? Cet appel a été entendu à propos de l'éditorial du numéro 4 de 2005, intitulé "Événements extrêmes : retours d'expérience ». Jean-Christophe Gaillard, géographe, insiste en effet sur la notion de "vulnérabilité » aux phénomènes extrêmes pour mettre l'accent sur le caractère éminemment social des catastrophes dites naturelles. Pour cet auteur, le changement des représentations sociales des causes des catastrophes pourrait déboucher sur une nouvelle approche de la gestion des risques. De son côté, Henri Décamps ${ }^{1}$, écologue, développe un aspect qui n'était qu'esquissé dans l'éditorial de 2005. Insistant sur la nature complexe des événements extrêmes et sur la difficulté d'identifier leurs causes, il préconise une analyse élargie de la vulnérabilité prenant en compte la résilience des systèmes socioécologiques. Ce faisant, il insiste sur le caractère nécessairement interdisciplinaire de toute démarche visant à se préparer aux événements extrêmes. Deux points de vue assez complémentaires, en réalité, d'un même problème de fond : celui du rôle des scientifiques confrontés aux « retours d'expérience » évoqués par notre éditorial du numéro 4 de 2005.
\end{abstract}

La Rédaction

\begin{abstract}
"Death may be the greatest equalizer, but when the Grim Reaper comes knocking only at the doors of the vulnerable, something is severely wrong." Catherine C. Liamzon, A Formula for Disaster, Panibagong Paraan, 2006².
\end{abstract}

\section{Le paradigme de l'extrême}

L'éditorial du volume 13, numéro 4, de Natures Sciences Sociétés s'interrogeait sur les événements extrêmes auxquels font habituellement référence les catastrophes dites «naturelles » ou « anthropiques » (Décamps et Mathieu, 2005). Ce texte faisait suite à plusieurs événements majeurs ayant frappé les esprits au travers d'une couverture médiatique sans précédent : le tsunami du 26 décembre 2004, 1'ouragan Katrina, le séisme

Auteur correspondant:

Jean-Christophe.gaillard@ujf-grenoble.fr

1 Voir, dans ce numéro, la contribution de H. Décamps «la vulnérabilité des systèmes socioécologiques aux événements extrêmes : exposition, sensibilité, résilience ».

2 Panibagong Paraan est un concours d'essais littéraires et académiques sur la question des inégalités, organisé en 2006 par la Banque mondiale aux Philippines. Le texte de C.C. Liamzon a remporté la compétition dans la catégorie «essais en anglais». au Pakistan. Percevoir les catastrophes liées à l'occurrence de phénomènes naturels comme des événements extrêmes est chose commune aussi bien dans les médias que dans le monde scientifique. Cette approche des catastrophes relève en effet d'un paradigme scientifique qui a monopolisé la littérature et influencé les politiques des institutions internationales pendant plus d'un demisiècle. Ce paradigme de l'extrême est magnifié dans l'ouvrage de I. Burton et al. (1993), The Environment As Hazard.

Envisager les catastrophes comme la résultante de phénomènes naturels "extrêmes ", en magnitude, et « rares », dans le temps, impose une gestion des risques apte à faire face à $1^{\prime}$ « extraordinaire ». Ces politiques relèvent bien souvent de mesures spécifiques et techniques : construire des digues pour parer à des crues exceptionnelles, mettre en place des systèmes de surveillance, envisager des plans d'alerte et d'évacuation. En parallèle, les sciences de la Terre s'attachent à mieux connaître et prévoir ces phénomènes exceptionnels, alors que la recherche en sciences humaines évalue la perception des phénomènes rares afin de comprendre les défaillances comportementales des populations - failles que doivent combler des campagnes d'information. L'ensemble de ces mesures répond à 
des logiques politiques (transfert de connaissance), économiques (transfert de technologie) et scientifiques (financements des programmes de recherche) très puissantes et globalisantes (Bankoff, 2001; Hewitt, 1995).

\section{Croissance du nombre de catastrophes et vulnérabilité}

Malgré les formidables progrès de la science, les moyens financiers considérables et les milliers de chercheurs mobilisés, le nombre de catastrophes augmente... Selon le Centre de recherche sur l'épidémiologie des désastres (CRED), le nombre de catastrophes liées à un phénomène naturel a décuplé entre les deux moitiés du XXe siècle, passant de 579 à 6723 événements (CRED, 2005). Si cette liste est sans doute incomplète pour les premières années de référence, elle traduit tout de même l'échec des politiques de gestion des risques et des catastrophes telles qu'envisagées durant ces cinquante dernières années. Cet échec tient très largement à l'inadéquation entre les politiques de $l^{\prime}$ « extrême » et la réalité des causes des catastrophes qui relèvent de contraintes quotidiennes matérialisées par la vulnérabilité des sociétés.

Les catastrophes touchent en premier lieu des laissés-pour-compte de l'ordinaire ou des oubliés du développement. Les victimes de catastrophes sont en effet vulnérables car elles sont marginalisées. Marginalisées géographiquement car elles habitent des secteurs menacés par des phénomènes naturels, marginalisées socialement car elles sont très souvent issues de groupes minoritaires, marginalisées économiquement parce qu'elles sont bien souvent pauvres, marginalisées politiquement car leur parole n'est pas reconnue sur la scène politique.

Un faisceau d'indicateurs cristallise la vulnérabilité des victimes de catastrophes (Cannon, 1994). Cellesci sont plus souvent des femmes (cas du tsunami du 26 décembre en Indonésie), des enfants et des personnes âgées (canicule de l'été 2003 en France), des handicapés, des prisonniers, des réfugiés. Les catastrophes frappent aussi plus souvent les individus aux revenus limités et fragiles (faibles salaires, emplois informels, absence d'épargne), qui limitent la protection contre les aléas naturels (localisation du domicile, type d'habitat, connaissance des moyens de protection). La vulnérabilité résulte également d'une protection sociale inadéquate (assurance santé, services de santé et d'éducation, retraites, normes de construction, mesures de prévention, etc.) et d'un capital social réduit (réseaux de solidarité).

\section{Vulnérabilité et contraintes structurelles}

La vulnérabilité s'inscrit dans des contraintes sociales, historiques et politico-économiques entremêlées (Fig. 1). Les contraintes sociales incluent le difficile accès à la terre et aux ressources d'une population en forte croissance. L'accès aux ressources conditionne la capacité à vivre dans des lieux sûrs et à construire un habitat résistant aux aléas naturels. En Asie et en Amérique latine, des milliers de paysans sans terre en quête de sécurité alimentaire s'installent sur des espaces exposés à des aléas naturels pour échapper à des systèmes agraires féodaux. Pour se nourrir au quotidien, on accepte de s'exposer à une éruption volcanique décennale ou à un typhon saisonnier plutôt que de reverser $75 \%$ de sa récolte aux propriétaires des bonnes terres. De même, les conflits armés et les guérillas forcent des milliers de victimes à s'établir dans des secteurs menacés par des phénomènes naturels. La menace $d^{\prime} u n$ tsunami «centennal » pèse moins lourd que la violence quotidienne. À ce titre, il n'est pas anodin que les deux régions les plus touchées par le tsunami du 26 décembre 2004 fussent frappées par des conflits armés multidécennaux (Aceh et la côte est du Sri Lanka).

Les déséquilibres d'accès aux ressources sont souvent hérités des époques coloniales, qui ont façonné les systèmes politiques de nombreux pays du Sud autour d'une élite resserrée contrôlant les activités économiques et le pouvoir, emprisonnant ainsi les victimes de catastrophes dans une situation de pauvreté chronique. L'orientation croissante de l'économie des pays du Sud vers les cultures d'exportation a également contribué à l'accroissement de la vulnérabilité en fragilisant l'accès aux ressources et en affaiblissant la sécurité alimentaire des victimes de catastrophes. Le cas des paysans du Sahel que l'on a poussés à délaisser leurs cultures de subsistance traditionnelles pour travailler dans des plantations fragiles face aux aléas climatiques est symptomatique (Copans 1975; Watts et Bohle, 1993).

Aux contraintes sociales et aux héritages historiques s'ajoute le contexte politico-économique contemporain. Les fluctuations du prix des matières premières sur les marchés internationaux ont des répercussions dramatiques pour nombre de petits agriculteurs exposés à des aléas naturels. En réponse à la chute des cours des produits agricoles, beaucoup de paysans des pays du Sud n'ont, par exemple, d'autres recours pour survivre que de se livrer à la coupe et au commerce illégal du bois, exposant ainsi leur communauté à des glissements de terrain. La dégradation environnementale et la vulnérabilité des sociétés sont aggravées par la prévalence d'intérêts particuliers ou étrangers au détriment des besoins collectifs ou locaux. Aux Philippines et en Indonésie, une poignée de multinationales, aidées par une élite locale complice, contrôlent l'exploitation des ressources naturelles tout en négligeant aléas naturels et vulnérabilité des populations (Bello et al., 2004). À l'échelle étatique, la corruption et le service de la dette, promulgué par la Banque mondiale et le Fond monétaire international, monopolisent une grande partie des budgets nationaux au détriment de la réduction de la vulnérabilité. 


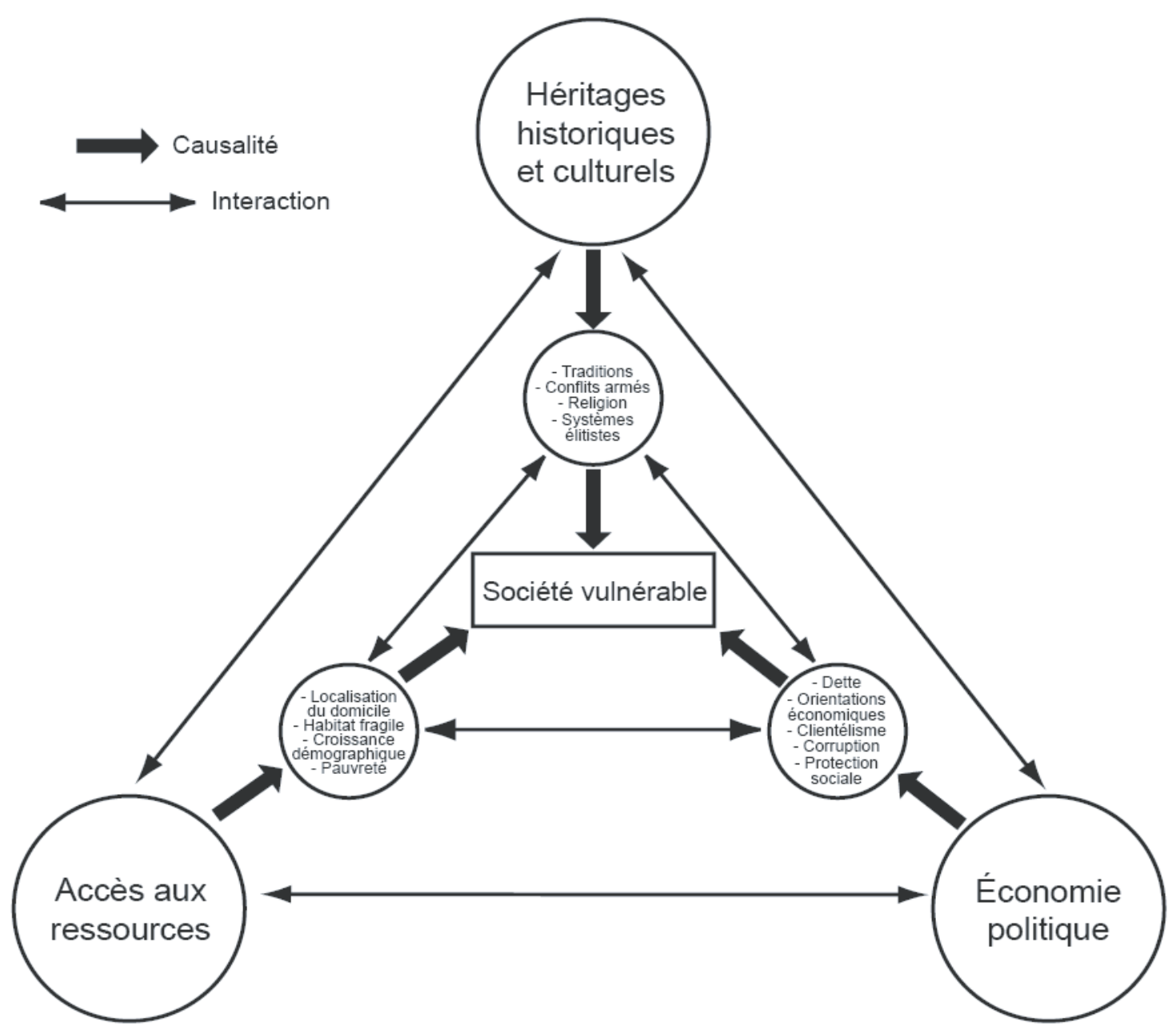

Fig. 1. Interactions et rapports de causalité entre facteurs de vulnérabilité.

Évidente dans les pays dits "en développement", cette démonstration vaut aussi pour les pays «industrialisés ». À titre d'exemple, l'origine de la catastrophe qui a frappé la Louisiane à la fin du mois d'août 2005 relève de la marginalisation territoriale, politique, sociale et économique quotidienne dont font l'objet les habitants de cette région, et non du caractère exceptionnel de l'ouragan Katrina, dont l'impact humain fut bien moindre en Floride. En France, on peut se demander qui sont les victimes de la canicule d'août 2003. Beaucoup de personnes âgées abandonnées à leur sort. Qui sont les victimes des vagues de froid hivernales? Les sans-abri exclus de la société. Et l'on pourrait étendre la démonstration à d'autres types de catastrophes et aux victimes de l'explosion de l'usine AZF, aux malades du sida, etc.

Les facteurs des catastrophes dépendent donc plus des contraintes quotidiennes qui piègent les victimes que de la dimension "extraordinaire » des aléas naturels. Dans cette logique, les catastrophes prolongent les situations d'urgence quotidienne pour les victimes et ne sont pas des accidents au sein de la société (Hewitt, 1983;
Maskrey, 1989; Wisner, 1993). Elles révèlent des lacunes en matière de développement, comme l'illustrent les famines du Sahel au cours des années 1970 et 1980 (Sen, 1983; Watts et Bohle, 1993). Dans ce contexte, faire porter la responsabilité des catastrophes à la nature consiste à se voiler la face, et le changement climatique actuel constitue, pour beaucoup de gouvernements et $\mathrm{d}$ 'institutions internationales, un alibi facile pour échapper à leur responsabilité dans la «vulnérabilisation » de la planète.

\section{Quotidien versus exceptionnel : pour une nouvelle approche de la gestion des risques}

De manière paradoxale, la vision dominante en matière de gestion des risques souligne l'« exceptionnel » de l'aléa et non le «quotidien » de la vulnérabilité. Parler de "politiques de gestion des risques et des catastrophes » équivaut donc à cloisonner les activités de prévention et de mitigation autour des aléas et à considérer les catastrophes comme des événements à part dans la société. 
Suivre cette logique conduit à dissocier les risques et les catastrophes du «quotidien » et, ainsi, à mésestimer les contraintes structurelles sous-jacentes. Parler de politique de gestion des risques et des catastrophes, c'est se limiter à traiter les symptômes tout en refoulant la racine du mal. Dès 1983, K. Hewitt (op. cit.) parlait à ce sujet $\mathrm{d}^{\prime}$ « archipel des désastres » et plaidait pour une remise en cause totale de l'approche contemporaine des catastrophes. Bien que relayé depuis (Wisner, 2001), cet appel a été peu entendu. Il apparaît pourtant que la réduction efficace $\mathrm{du}$ risque passe par une action en amont de la seule prévention des phénomènes naturels "extrêmes » et "rares", au travers de la réduction de la pauvreté, d'un accès égalitaire aux ressources et d'une protection sociale et sociétale adaptée. Ces considérations renvoient aux politiques de développement et d'aménagement du territoire au sens large, et non à des politiques de gestion des risques déconnectées de leur contexte socioéconomique. Elles font enfin et surtout appel au respect des droits de l'homme.

\section{Références}

Bankoff, G., 2001. Rendering the world unsafe: 'vulnerability' as western discourse, Disasters, 25, 1, 19-35.

Bello, W., Docena, H., de Guzman, M., Malig, M., 2004. The AntiDevelopmental State: The Political Economy of Permanent Crisis in the Philippines, Quezon City, University of the Philippines Department of Sociology/Focus on the Global South.
Burton, I., Kates, R.W., White, G.F., 1993 (2nd ed.). The Environment As Hazard, New York, The Guilford Press.

Cannon, T., 1994. Vulnerability analysis and the explanation of 'natural' disasters, in Varley, A. (Ed.), Disasters, Development and Environment, Chichester, John Wiley \& Sons, 13-30.

Copans, J. (Ed.), 1975. Sécheresses et Famines du Sahel, Paris, Maspero.

CRED, 2005. EM-DAT: the OFDA/CRED International Disaster Database, Louvain, Université catholique de Louvain, http://www.cred.be/emdat (consulté le 31 mars 2005).

Décamps H., Mathieu N. 2005. Événements extrêmes : retours d'expérience, Natures Sciences Sociétés, 13, 4, 369-370.

Hewitt, K., 1983. The idea of calamity in a technocratic age, in Hewitt, K. (Ed.), Interpretation of Calamities, Boston, Allen \& Unwin Inc., 3-32.

Hewitt, K., 1995. Sustainable disasters? Perspectives and powers in the discourse of calamity, in Crush, J. (Ed.), Power of Development, London, Routledge, 115-128.

Maskrey, A., 1989. Disaster Mitigation: A Community-Based Approach, Oxford, Oxfam.

Sen, A.K., 1983. Poverty and Famines: An Essay on Entitlement and Deprivation, Oxford, Oxford University Press.

Watts, M.J., Bolhe, H.G., 1993. The space of vulnerability: the causal structure of hunger and famine, Progress in Human Geography, 17, 1, 43-67.

Wisner, B., 1993. Disaster vulnerability: scale, power, and daily life, Geojournal, 30, 2, 127-140.

Wisner, B., 2001. Changes in capitalism and global shifts in the distribution of hazard and vulnerability, in Pelling, M. (Ed.), Natural Disasters and Development in a Globalizing World, London, Routledge, 43-56. 